\title{
Deletion of Mitochondrial DNA in a Case of Early-Onset Diabetes Mellitus, Optic Atrophy, and Deafness (Wolfram Syndrome, MIM 222300)
}

\author{
Agnès Rötig, Valérie Cormier, Pierre Chatelain, * René Francois, * Jean-Marie Saudubray, Pierre Rustin, and Arnold Munnich \\ Unité de Recherches sur les Handicaps Génétiques de l'Enfant INSERM U12 and Département de Pédiatrie, Hôpital des Enfants- \\ Malades, 75743 Paris Cedex 15, France; and *Service d'Endocrinologie et Diabétologie Pédiatrique, \\ Hôpital Edouard Herriot, 69437 Lyon Cedex 03, France
}

\begin{abstract}
The Wolfram syndrome (MIM 222300) is a disease of unknown origin consisting of diabetes insipidus, diabetes mellitus, optic atrophy, and deafness. Here we report on a generalized deficiency of the mitochondrial respiratory enzyme activities in skeletal muscle and lymphocyte homogenate of a girl suffering from the Wolfram syndrome. In addition, we provide evidence for a 7.6-kilobase pair heteroplasmic deletion (spanning nucleotides 6465-14135) of the mitochondrial DNA in the two tissues and show that directly repeated sequences ( $11 \mathrm{bp}$ ) were present in the wild-type mitochondrial genome at the boundaries of the deletion. Neither of the patient's parents was found to bear rearranged molecules.

This study supports the view that a respiratory chain defect can present with insulin-dependent diabetes mellitus as the onset symptom. It also suggests that a defect of oxidative phosphorylation should be considered when investigating other cases of Wolfram syndrome, especially because this syndrome fulfills the criteria for a genetic defect of the mitochondrial energy supply: ( $a$ ) an unexplained association of symptoms $(b)$ with early onset and rapidly progressive course, $(c)$ involving seemingly unrelated organs and tissues. (J. Clin. Invest. 1992. 91:1095-1098.) Key words: deafness $\bullet$ growth hormone neurosecretory dysfunction • mitochondrial DNA deletion • mitochondrial respiratory chain $\bullet$ type 1 diabetes $\bullet$ Wolfram syndrome
\end{abstract}

\section{Introduction}

Since the original report by Luft et al. (1), mitochondrial disorders have been exclusively regarded as neuromuscular diseases. In fact, oxidative phosphorylation not only takes place in muscles and nerves but is present in other tissues as well, inasmuch as the oxidation of fuel molecules by oxygen and the concomitant energy transduction into ATP also occur in a number of non-neuromuscular organs, via four multienzymatic complexes: NADH-coenzyme $\mathrm{Q}(\mathrm{CoQ})$ reductase (complex I), succinate $\mathrm{CoQ}$ reductase (complex II), CoQ-H2 cytochrome $c$ reductase (complex III), and cytochrome $c$ oxidase (complex IV).

Address reprint requests to Dr. Arnold Munnich, Hôpital des EnfantsMalades, Tour Technique Lavoisier (2ème étage), 149, rue de Sèvres, 75743 Paris Cedex 15, France.

Received for publication 24 March 1992 and in revised form 23 July 1992.

J. Clin. Invest.

(c) The American Society for Clinical Investigation, Inc.

0021-9738/93/03/1095/04 \$2.00

Volume 91, March 1993, 1095-1098
Consequently, a disorder of oxidative phosphorylation might theoretically give rise to any symptom in any organ or tissue. In addition, any mode of inheritance could be expected, owing to the twofold genetic origin of mitochondrial respiratory enzymes that are encoded both by the nuclear genome and by the mitochondrial DNA (mtDNA) ${ }^{1}$ a maternally inherited 16.5-kilo base pair ( $\mathrm{kbp}$ ) circular genome (2).

In support of this, we have previously shown that genetic defects of the mitochondrial energy supply can present with pancytopenia (3), proximal tubulopathy (4), or hepatic failure as the onset symptoms (5).

Here we report on a severe respiratory enzyme deficiency in a patient with the Wolfram syndrome-diabetes insipidus, diabetes mellitus, optic atrophy, and deafness (DIDMOAD), MIM 222300 (6) -and show that a mitochondrial disorder can present with diabetes mellitus as the onset symptom. In addition, we describe the deletion of the mitochondrial genome between directly repeated sequences as the possible cause of the disease in the patient. Based on this observation, we hypothesize that disorders of mitochondrial energy supply could possibly account for other cases of Wolfram syndrome as well as for several syndromes of hitherto unknown origin.

\section{Case report}

A girl was born after a term pregnancy to unrelated healthy parents. She was small for gestational age for no obvious reason (birthweight $2,800 \mathrm{~g}$, height $45 \mathrm{~cm}$, head circumference $33 \mathrm{~cm}$ ) but she did relatively well during the first few months of life: she could sit unaided at $9 \mathrm{mo}$, walk at $13 \mathrm{mo}$, and speak at 24 mo. At $1 \mathrm{yr}$ of age, however, asthenia, loss of weight $(1.3 \mathrm{~kg})$, polyurodypsy, and glycosuria led to the discovery of type 1 diabetes mellitus requiring insulin administration. Her HLA haplotype did not provide evidence for susceptibility to insulin-dependent diabetes mellitus (DR 11-14, DP 402-301). Her condition markedly improved thereafter but she progressively developed bilateral optic atrophy and sensorineural hearing loss (60-db decay for higher frequencies). For this reason, Wolfram syndrome (DIDMOAD, MIM 222300) was diagnosed. During her sixth year, however, her condition markedly worsened and she gradually developed failure to thrive, cerebellar ataxia, night blindness, progressive external ophthalmoplegia, extrapyramidal syndrome, and mental retardation with elevated protein levels in the cerebral spinal fluid (1-1.5 $\mathrm{g} /$ liter, normal $<0.30$ ) and low-density areas in peduncles and putamen on nuclear magnetic resonance examination of the brain.

1. Abbreviations used in this paper: DIDMOAD, diabetes insipidus, diabetes mellitus, optic atrophy, and deafness; mtDNA, mitochondrial DNA; nt, nucleotide. 
Although diabetes was adequately controlled under Umulin and Umulin NPH administration (Eli Lilly \& Co., Indianapolis, IN), she presented recurrent nocturnal hypoglycemias between midnight and 7 a.m. (blood glucose $<30 \mathrm{mg} /$ liter). Plasma C-peptide was negative and careful blood glucose monitoring did not allow one to relate hypoglycemic episodes to either excessive insulin dosage or insufficient food intake. Because she had a proportionate growth failure (height below 3 $\mathrm{SD}$ ) and a delayed bone age of unexplained origin, she was investigated for a possible growth hormone $(\mathrm{GH})$ deficiency. Plasma immunoreactive GH concentration was markedly reduced (mean plasma immunoreactive GH was $2 \mathrm{ng} / \mathrm{ml}$ between 8 p.m. and 7 a.m.; three plasma peaks of 9.4, 7.6, and $4.3 \mathrm{ng} / \mathrm{ml}$, blood sampling every $20 \mathrm{~min}$ ). By contrast, peak plasma GH was observed after either growth releasing factor administration $(20 \mathrm{ng} / \mathrm{ml})$ or a propranolol + glucagon provocative test $(19.2 \mathrm{ng} / \mathrm{ml})$. Thyroid status was normal. This condition was consistent with GH neurosecretory dysfunction (7). For this reason, human recombinant $\mathrm{GH}$ administration was started ( $2 \mathrm{IU} / \mathrm{d}$, subcutaneously), but her neurological condition markedly worsened shortly after $\mathrm{GH}$ administration was started.

At $13 \mathrm{yr}$ of age, she has major growth retardation (below 3 SD) and severe amyotrophy and has lost the ability to stand and walk. She has bilateral deafness, extrapyramidal syndrome, bilateral absence of deep tendon reflexes of the inferior limbs, total blindness, retinitis pigmentosa, and major difficulties in swallowing. The course of her diabetes is unremarkable (hemoglobin Alc 6-7.2\%; normal \pm SD $5.2 \pm 0.4$ ) and the requirement for insulin is moderate $(0.8 \mathrm{U} / \mathrm{kg}$ per day $)$. No evidence for heart, kidney, or skin involvement has been hitherto noted. However, she has dry thick and brittle hair and repeated episodes of erythrocyanosis involving toe and finger extremities have been noted.

\section{Methods}

Plasma lactate/pyruvate and ketone body molar ratios (3-OH-butyrate/acetoacetate) were determined in the patient and in controls as indexes of oxidation-reduction status in cytoplasm and mitochondria, respectively. Biopsy specimens of the deltoid were taken under local anesthesia and spectrometric analyses of mitochondrial respiratory enzymes in skeletal muscle and lymphocyte homogenates were carried out as described (8).

For Southern blotting, total DNA ( $5 \mu \mathrm{g}$ ) derived from muscle or lymphocytes was digested, separated by agarose gel $(0.7 \%)$ electropho- resis and transferred onto nylon filters $\left(\right.$ Hybond $\mathrm{N}^{+}$, Amersham International, Amersham, UK). The filters were hybridized with $\left[{ }^{32} \mathrm{P}\right]-$ dCTP-labeled mtDNA probes (cytochrome $b$ or NADH dehydrogenase $4,2 \times 10^{6} \mathrm{cmp} / \mathrm{ml}$ ). For characterization of the nucleotide sequence at the boundaries of the deletion, $1 \mu \mathrm{g}$ total DNA was submitted to polymerase chain reaction (PCR) amplification ( 30 cycles) with two oligonucleotide primers ( $p$ rimer $\mathrm{A}=$ nucleotides ( $\mathrm{nt}$ ) 5881-5901, primer $B=n t 14236-14257$ ). DNA was amplified by mixing $100 \mathrm{pmol}$ of each primer with $2.5 \mathrm{U}$ of Taq polymerase (Perkin Elmer Cetus, Norwalk, CT) as described (9). Amplified DNA was recovered on agarose gel, then directly sequenced by the Sanger technique, and compared to the wild-type sequence (10).

\section{Results}

Lactate levels and lactate/pyruvate molar ratios in plasma were slightly and transiently elevated in the postabsorptive period (plasma levels in the fed patient $1.7-3.5 \mathrm{mmol} /$ liter, normal $<2.4$; lactate/pyruvate molar ratios $13-18$, normal $<20$ ). These results prompted us to carry out enzyme assays on skeletal muscle and lymphocyte homogenates and to finally identify a generalized deficiency of respiratory enzyme activities in both tissues (Table I). In addition, ratios that best account for balanced respiratory enzyme activities in vivo (11) were in the normal range, a feature that could be related to the concomitant deficiency of several enzyme activities (Table I).

Southern blot analysis of skeletal muscle DNA digested with restriction enzyme BamHI (cleavage at position 14258) and hybridization using a cytochrome $b$ probe showed that the patient had two populations of mtDNA, one normal $(16.5 \mathrm{~kb})$ and one partly deleted ( $9 \mathrm{~kb}$, Fig. 1, lane 1$)$, which is not detected using a NADH dehydrogenase 4 probe (Fig. 1, lane 3 ). Interestingly, the same pattern of heteroplasmy was found in her lymphocytes (Fig. 1, lane 2). PCR amplification and sequence analysis carried out to characterize the end points of the rearrangement showed that the deletion spanned $7,670 \mathrm{bp}$, from nt6465 to nt 14135 and skipped the genes for four subunits of complex I (ND3, ND4L, ND4, and the major part of ND5), three subunits of complex IV (COXII, COXIII, part of COXI), two subunits of complex V (ATPase 6, ATPase 8), and 8 tRNAs (Fig. 1, right). Sequence analysis also showed that the abnormal junction was located between COXI and ND5 and resulted in an in-frame COXI-ND5 chimeric gene (Fig. 1, bottom). Interestingly, two directly repeated sequences of $11 \mathrm{bp}$ were present in the wild-type mitochondrial genome at the boundaries of the deletion (Fig. 1, bottom). Finally, none

Table I. Enzyme Activities in Circulating Lymphocytes and Muscle Tissue

\begin{tabular}{|c|c|c|c|c|c|}
\hline \multirow[b]{3}{*}{ Tissues } & \multirow{2}{*}{\multicolumn{3}{|c|}{ Enzyme activities }} & \multicolumn{2}{|c|}{ Ratios } \\
\hline & & & & \multirow{2}{*}{$\begin{array}{l}\text { COX/ } \\
\text { SCCR }\end{array}$} & \multirow{2}{*}{$\begin{array}{l}\text { SCCR/ } \\
\text { NCCR }\end{array}$} \\
\hline & $\operatorname{cox}^{\prime}$ & SCCR & NCCR & & \\
\hline \multicolumn{6}{|c|}{ nmol $\mathrm{min}^{-1} \mathrm{mg}^{-1}$ protein } \\
\hline \multicolumn{6}{|l|}{ Peripheral lymphocytes } \\
\hline Patient & 50 & 16 & - & 3.1 & - \\
\hline Control range $(n=10)$ & $69-146$ & $28-46$ & - & $3.1 \pm 0.5^{*}$ & - \\
\hline \multicolumn{6}{|l|}{ Skeletal muscle } \\
\hline Patient & 34 & 9 & 14 & 3.8 & 0.6 \\
\hline Control range $(n=35)$ & $56-654$ & $18-184$ & $15-288$ & $3.4 \pm 0.4 *$ & $1.0 \pm 0.2$ \\
\hline
\end{tabular}

Abbreviations: COX: Cytochrome $c$ oxidase; SCCR: succinate cytochrome $c$ reductase; NCCR: rotenone-sensitive NADH cytochrome $c$ reductase. * Mean value \pm 1 SD. 

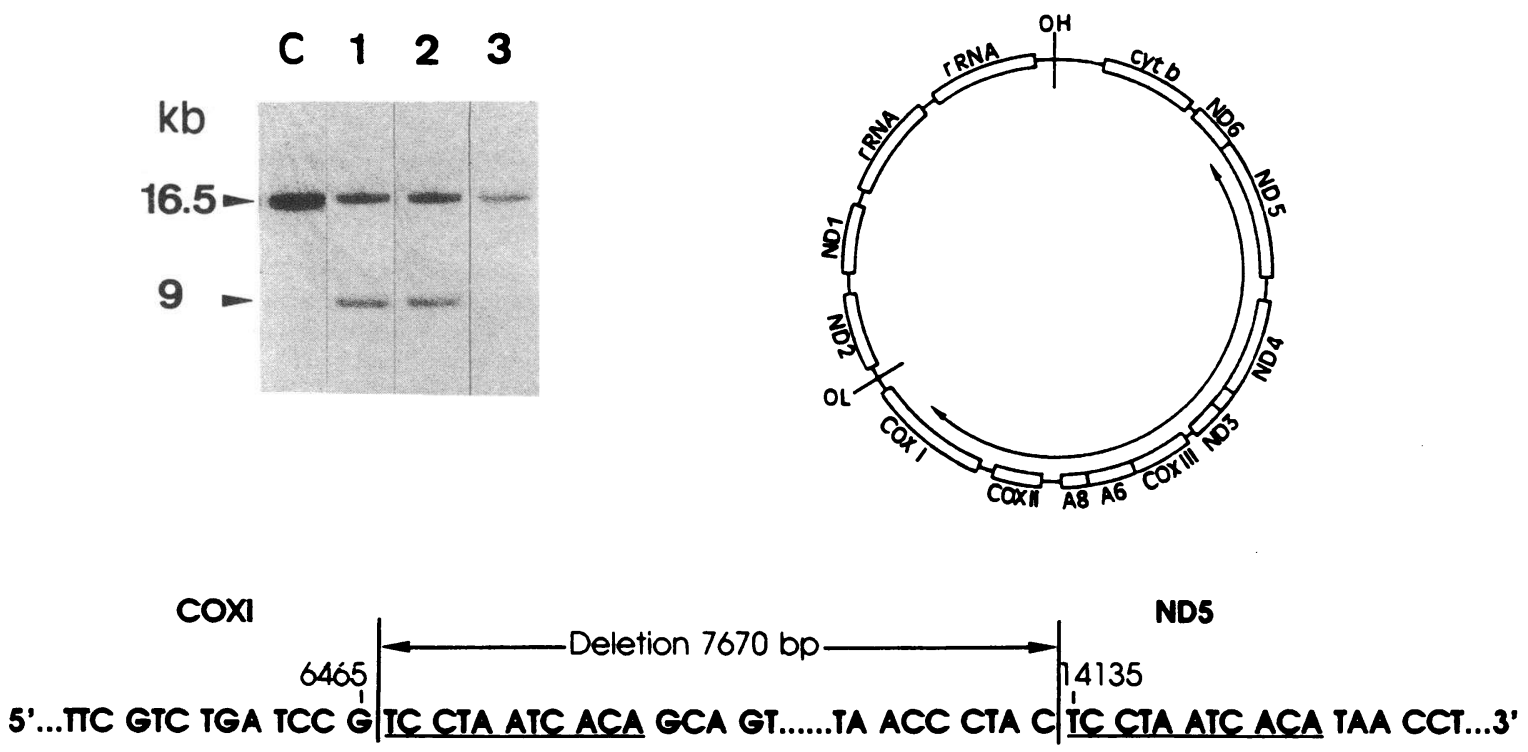

Figure 1. (Left) Hybridization pattern of the mtDNA probes in patient's lymphocytes (lane 2) and muscle DNA (lanes 1 and 3 ) and in control DNA $(C)$ digested with restriction enzyme BamHI. Normal ( $16.5 \mathrm{~kb})$ and 9-kb abnormal BamHI fragments were detected using a cytochrome $b$ probe (lanes 1 and 2 ) and the normal fragment only was detected using a NADH dehydrogenase 4 probe (lane 3). (Right) Map of human mtDNA showing the extent of the deletion in the patient. (Bottom) Characterization of the nucleotide sequence at the boundaries of the mtDNA deletion. A 11-bp direct repeat in the wild-type mtDNA (underlined) was found to flank the 7,670-bp deletion in the patient.

of the patient's parents were found to bear rearranged molecules even when their lymphocyte DNA was submitted to PCR amplification using specific oligomers (not shown).

\section{Discussion}

The present study reports on a heteroplasmic deletion of the mitochondrial genome in a patient with insulin-dependent diabetes mellitus in early infancy. Because she gradually developed hearing loss and optic atrophy, she was first considered to have Wolfram syndrome (MIM 222300) which consists of DIDMOAD (6). Despite the latency of diabetes insipidus, the diagnosis of Wolfram syndrome was not originally rejected and only at the point when progressive cerebellar and cortical involvement occurred could the hypothesis of a genetic defect of oxidative phosphorylation be considered. The progressive organ involvement and the observation of a mild hyperlactatemia pointed to a possible disorder of the mitochondrial energy supply and finally led us to identify a generalized deficiency of the respiratory chain and a heteroplasmic deletion of the mtDNA. Thus, early diagnosis was difficult, especially as no familial or genetic factors predisposed to the disease. Later on, however, diagnosis was easier when seemingly unrelated symptoms occurred, namely optic atrophy and deafness. Based on this 'observation, we suggest that a possible defect of oxidative phosphorylation should be considered when investigating other cases of Wolfram syndrome, especially as this syndrome fulfills the criteria for a genetic defect of the mitochondrial energy supply: (a) an unexplained association of symptoms, (b) with early onset and a rapidly progressive course, $(c)$ involving seemingly unrelated organs that share no common embryological origin or biological function (12).

This observation along with those of Poulton et al. (13), Majander et al. (14), and Ballinger et al. (15) is, to our knowledge, the first report of a mitochondrial disorder presenting with insulin-dependent diabetes mellitus as the onset symptom.
Yet, how diabetes mellitus is related to the mtDNA deletion and why the endocrine pancreas was the first targeted tissue remain intriguing. Bearing in mind that the rate of the glucose oxidation controls insulin secretion and release in $\beta$ cells, one should consider a defective insulin response to glucose stimulation as a consequence of the respiratory chain deficiency in the pancreas $(16,17)$. In this case, random partitioning of mitochondria during development might have resulted in the fortuitous accumulation of deleted mtDNA-containing mitochondria in particular tissues including pancreas. It is also tempting to speculate that the mtDNA deletion gave rise to an anti- $\beta$-islet antibody response via the stimulation of the autoimmune system by a mitochondrial antigene as already demonstrated in mice (18) and in humans as well (19). Indeed, one could hypothesize that the rearrangement reported here produced a potentially antigenic chimeric protein containing a COXI moiety joined to a NADH dehydrogenase 5 (ND5) moiety, in that the deletion did not suppress the reading frame of the rearranged mitochondrial genome. Hitherto, however, no evidence for anti- $\beta$-islet antibodies has been found in the plasma of our patient (not shown).

Why a deletion of the mtDNA resulted in a severe respiratory chain dysfunction is also intriguing, especially inasmuch as affected tissues contained deleted and nondeleted mtDNA molecules (heteroplasmy). One possible explanation is that the chimeric protein competed with the normal counterpart and altered the function of the corresponding complexes. Alternatively, the mitochondrially encoded subunits, whose genes are spanned by the 7.6-kb mtDNA deletion, could be rate limiting, especially in that a generalized deficiency of respiratory enzyme activities was observed both in skeletal muscle and in peripheral lymphocytes.

We and others have previously described an elevated oxidation-reduction status in plasma as a useful clue to the diagnosis of respiratory chain deficiency $(4,20)$. The rationale for this is that the impaired respiratory chain accumulates NADH instead of transferring reducing equivalents to oxygen. This re- 
sults in the accumulation of NADH and the lack of NAD, with the secondary impairment of the Krebs cycle and subsequent elevation of lactate and lactate/pyruvate and ketone body molar ratios in plasma. This is particularly true in the postabsorptive period when more NAD is required for adequately metabolizing glycolytic substrates. In the present study, however, lactate levels were moderately elevated and the lactate/pyruvate ratios remained in the normal range, even in the fed patient. This feature could be related to diabetes mellitus which hampers entry of pyruvate into the Krebs cycle. We have made similar observations in patients with mitochondrial cytopathies who later developed diabetes mellitus in the course of their diseases (4). It is therefore important to be aware that insulinopenia can be a pitfall in the investigation of the oxidation-reduction status in vivo, especially because the endocrine pancreas is frequently involved in the course of these diseases (12).

It is worth noting also that the case of Wolfram syndrome reported here was sporadic, while mitochondria are known to be maternally inherited in man (21). The absence of either affected relatives or maternal inheritance of the rearrangement is suggestive of a de novo mutation. In addition, the direct repeats found at the boundaries of the deleted sequence might have promoted intramolecular recombinations of the mitochondrial genome via either replication slippage or breakage reunion. Whatever the mechanism, it is worth remembering that deletions of the mitochondrial genome between directly repeated sequences have now been reported in a variety of strikingly different conditions, including Kearns-Sayre syndrome (22), mitochondrial myopathies (23), and Pearson's marrowpancreas syndrome (3).

A large spectrum of clinical outcomes and concomitant symptoms have been reported in the Wolfram syndrome: some cases responded to thiamine (24) whereas others were associated with malformations (brachydactyly and hydronephrosis) $(25,26)$. It is therefore obvious that, although the present case has been ascribed to a defect of the mitochondrial energy supply, not all cases of Wolfram syndrome are accounted for by this mechanism. Indeed, this case supports the view that the Wolfram syndrome includes a variety of clinically and genetically heterogeneous conditions.

In conclusion, it appears that a mitochondrial cytopathy can present with insulin-dependent diabetes mellitus as the onset symptom. The diagnosis of mitochondrial disorder can hardly be considered early, especially when the onset symptom is the only one present. By contrast, this diagnosis is a more obvious possibility when seemingly unrelated symptoms occur as in this case of Wolfram syndrome. Based on this observation and bearing in mind the ubiquitous nature of oxidative phosphorylation, we would suggest giving consideration to genetic defects of the mitochondrial energy supply in elucidating the origin of other diseases with seemingly unrelated symptoms.

\section{Acknowledgments}

We are thankful to Monique Poussière, Gérard Reach, and Alan Strickland for their contribution to this manuscript and to the Association Française contre les Myopathies (A.F.M.) for financial support.

\section{References}

1. Luft, R., D. Ikkos, and G. Palmieri. 1962. Severe hypermetabolism of non thyroid origin with a defect in the maintenance of mitochondrial respiratory control: a correlated clinical, biochemical and morphological study. J. Clin. Invest. $41: 1776-1804$

2. Wallace, D. 1989. Mitochondrial DNA mutations and neuromuscular disease. Trends Genet. 5:9-13.

3. Rötig, A., V. Cormier, S. Blanche, J. P. Bonnefont, F. Ledeist, N. Romero, J. Schmitz, P. Rustin, A. Fischer, J. M. Saudubray, et al. 1989. Pearson's marrowpancreas syndrome: a multisystem mitochondrial disorder in infancy. J. Clin. Invest. 86:1601-1608.

4. Rötig, A., J. L. Bessis, N. Romero, V. Cormier, J. M. Saudubray, P. Narcy, G. Lenoir, P. Rustin, and A. Munnich. 1992. Maternally inherited duplication of the mitochondrial genome in a syndrome of proximal tubulopathy, diabetes mellitus and cerebellar ataxia. Am. J. Hum. Genet. 50:364-370.

5. Cormier, V., P. Rustin, J. P. Bonnefont, C. Rambaud, S. Couderc, F. Parrot-Roulaud, M. Carré, J. C. Risse, C. Cahuzac, J. M. Saudubray, et al. 1991. Hepatic failure in neonatal-onset disorders of oxidative phosphorylation. $\mathrm{J}$. $\mathrm{Pe}$ diatr. 119:951-954.

6. Wolfram, D. J., and H. P. Wagener. 1938. Diabetes mellitus and simple optic atrophy among siblings: report of four cases. Mayo Clin. Proc. 13:715-718.

7. Spiliotis, B., G. August, W. Mung, W. Sonis, W. Mendelson, and B. Bercu. 1984. Growth hormone neurosecretory dysfunction. JAMA (J. Am. Med. Assoc.). 251:2223-2230.

8. Chrétien, D., T. Bourgeron, A. Rötig, A. Munnich, and P. Rustin. 1990. The measurement of the rotenone sensitive NADH cytochrome $c$ reductase activity in mitochondria isolated from minute amount of human skeletal muscle. Biochem. Biophys. Res. Commun. 173:26-33.

9. Ehrlich, H. A., D. H. Gelfand, and R. K. Saiki. 1988. Specific DNA amplification. Nature (Lond.). 331:461-462.

10. Anderson, S., A. T. Bankier, B. G. Barrell, M. H. L. de Bruijn, A. R. Coulson, J. Drouin, I. C. Eperon, N. P. Nierlich, B. A. Roe, F. Sanger, et al. 1981. Sequence and organization of the human mitochondrial genome. Nature (Lond.). 290:457-465.

11. Rustin, P., D. Chretien, T. Bourgeron, A. Rötig, and A. Munnich. 1991. Assessment of the mitochondrial respiratory chain. Lancet. ii: 60.

12. Munnich, A., P. Rustin, A. Rötig, D. Chrétien, J. P. Bonnefont, C. Nuttin V. Cormier, A. Vassault, P. Parvy, J. Bardet, et al. 1992. Clinical aspects of mitochondrial disorders. J. Inherited Metab. Dis. 15:448-455.

13. Poulton, J., M. E. Deadman, and R. M. Gardiner. 1989. Duplications of mitochondrial DNA in mitochondrial myopathy. Lancet. i:236-240.

14. Majander, A., A. Suomalainen, K. Vettenranta, H. Sariola, M. Perkkiö, C. Holmberg, and H. Pihko. 1991. Congenital hypoplastic anemia, diabetes, a severe renal dysfunction associated with a mitochondrial DNA deletion. Pediatr. Res. 30:327-330.

15. Ballinger, S. W., J. M. Shoffner, E. V. Hedaya, I. Trounce, M. A. Polak, D. A. Koontz, and D. C. Wallace. 1992. Maternally transmitted diabetes and deafness associated with a $10.4 \mathrm{~kb}$ mitochondrial DNA deletion. Nature Genet. $1: 11-15$.

16. Coudé, F. X., H. Ogier, A. Munnich, C. Marsac, C. Charpentier, and J. M. Saudubray. 1982. Defective insulin response to intravenous glucose in congenital lactic acidosis. Pediatr. Res. 16:85.

17. Malaisse, W. J. 1992. Insulin biosynthesis and secretion in vitro. In International Textbook of Diabetes Mellitus. K. G. M. M. Alberti, editor. John Wiley \& Sons, Inc., New York. 261-284.

18. Loveland, B., C. R. Wang, H. Yonekawa, E. Hermel, and K. Fischer-Lindahl. 1990. Maternally transmitted histocompatibility antigen of mice: a hydrophobic peptide of a mitochondrially encoded protein. Cell. 60:971-980.

19. Schapira, A. H., J. M. Cooper, D. Dexter, J. B. Clark, P. Jenner, and C. D. Marsen. 1990. A mitochondrial encephalomyopathy with specific deficiencies of two respiratory chain polypeptides and a circulating autoantibody to a mitochondrial matrix protein. Brain. 113:419-432.

20. Robinson, B. H. 1989. Lactic acidemia. In The Metabolic Basis of Inherited Diseases, 6th edition. C. R. Scriver, A. L. Beaudet, W. S. Sly and D. Valle, editors. McGraw-Hill, Inc., New York. 869-888.

21. Giles, R. E., H. Blanc, H. M. Cann, and D. C. Wallace. 1980. Maternal inheritance of human mitochondrial DNA. Proc. Natl. Acad. Sci. USA. 77:67156719.

22. Lestienne, P., and G. Ponsot. 1988. Kearns-Sayre syndrome with muscle mitochondrial DNA deletion. Lancet. i:885.

23. Holt, I. J., A. E. Harding, and J. A. Morgan-Hughes. 1988. Deletion of muscle mitochondrial DNA in patients with mitochondrial myopathies. Nature (Lond.). 331:717-719.

24. Borgna-Pignatti, C., P. Marradi, L. Pinelli, N. Monetti, and C. Patrini. 1989. Thiamine-responsive anemia in DIDMOAD syndrome. J. Pediatr. 114:405-410.

25. Bale, A. E., I. H. Ludwig, L. A. Effron, and Z. N. Zakov. 1985. Linkage between the genes for Wolfram syndrome and brachydactyly. Am. J. Med. Genet. 20:733-734.

26. Salih, M. A. M., and T. Tuvemo. 1991. Diabetes insipidus, diabetes mellitus, optic atrophy and deafness (DIDMOAD syndrome): a clinical study of two Sudanese families. Acta Paediatr. Scan. 80:567-572. 